\title{
Eficácia da máscara facial (TNT) na população para a prevenção de infecções por coronavírus: revisão sistemática
}

\author{
Effectiveness of the use of non-woven face mask to prevent \\ coronavirus infections in the general population: a rapid systematic \\ review
}

\begin{abstract}
Maria Cristina de Camargo (https://orcid.org/0000-0001-9615-655X) ${ }^{1}$
Martha Sílvia Martinez-Silveira (https://orcid.org/0000-0002-1004-1784) ${ }^{2}$

Adeânio Almeida Lima (https://orcid.org/0000-0003-1171-8299) ${ }^{3}$

Bruno Pires Bastos (https://orcid.org/0000-0003-2778-8056) ${ }^{4}$

Diana Lima dos Santos (https://orcid.org/0000-0002-9246-0228) ${ }^{5}$

Sara Emanuela de Carvalho Mota (https://orcid.org/0000-0001-9502-5646) ${ }^{6}$

Roberta Borges Silva (https://orcid.org/0000-0001-7273-5151) ${ }^{7}$

Isabela Porto de Toledo (https://orcid.org/0000-0003-2958-3265) ${ }^{8}$
\end{abstract}

${ }^{1}$ Universidade Estadual de Feira de Santana. Av. Transnordestina s/n, Novo Horizonte. 44036-900 Feira de Santana BA Brasil. mariacristinac77@

gmail.com

${ }^{2}$ Instituto Gonçalo Moniz, Fiocruz. Salvador BA Brasil.

${ }^{3}$ Secretaria de Saúde de Inhambupe. Inhambupe BA Brasil.

${ }^{4}$ Núcleo de

Desenvolvimento Integrado de Produtos, Universidade Federal de Santa Catarina. Florianópolis SC Brasil. ${ }^{5}$ Instituto de Saúde

Coletiva, Universidade Federal da Bahia. Salvador BA Brasil.

${ }^{6}$ Superintendência Estadual do Ministério da Saúde na Bahia. Salvador BA Brasil. ${ }^{7}$ Departamento de Ciência e Tecnologia, Ministério da Saúde. Brasília DF Brasil.

${ }^{8}$ Universidade de Brasília.

Brasília DF Brasil.

\begin{abstract}
Objectives: to evaluate the effectiveness of non-woven face masks for the prevention of respiratory infections (MERS CoV, SARS-CoV, and SARS-CoV-2) in the population. Methods: search in Medline, Embase, Cinahl, The Cochrane Library, Trip databases. Google Scholar, Rayyan and medRxiv were also consulted for complementary results. No filters related to date, language or publication status were applied. Titles and abstracts were screened, and later, full texts were evaluated. Results: three studies were included: $a$ randomized cluster clinical trial and two systematic reviews. The clinical trial indicates a potential benefit of medical masks to control the source of clinical respiratory disease infection. In one of the systematic reviews, it was not possible to establish a conclusive relationship between the use of the mask and protection against respiratory infection. Finally, another systematic review indicated that masks are effective in preventing the spread of respiratory viruses. Conclusion: Evidence points to the potential benefit of standard non-woven face masks. For the current pandemic scenario of COVID-19, education on the appropriate use of masks associated with individual protection measures is recommended.
\end{abstract}

Key words Coronavirus of Middle East Respiratory Syndrome, Severe acute respiratory syndrome, Coronavirus, Masks, Prevention
Resumo O objetivo deste artigo é avaliar a eficácia das máscaras faciais padrão tecido não tecido (TNT) para a prevenção de doenças respiratórias (MERS CoV, SARS-CoV e SARS-CoV-2) na população. Foi realizada busca nas bases de dados Medline, Embase, Cinahl, The Cochrane Library, Trip. Também busca complementar no Google Acadêmico, Rayyan e medRxiv. Não foram aplicados filtros relacionados a data, idioma ou status de publicação. Títulos e resumos foram rastreados $e$, posteriormente, textos completos foram avaliados. Foram incluídos três estudos: um ensaio clínico randomizado tipo cluster e duas revisões sistemáticas. O ensaio clínico indica benefício potencial de máscaras médicas para controle da fonte de infecção, para a doença respiratória clínica. Em uma das revisões sistemáticas, não foi possivel estabelecer relação conclusiva entre uso da máscara e proteção contra infecção respiratória. Por fim, outra revisão sistemática demonstrou que máscaras são eficazes na prevenção da propagação de vírus respiratórios. As evidências apontam para benefício potencial das máscaras faciais padrão TNT. Para o cenário atual de pandemia por COVID 19, recomenda-se educação sobre uso adequado de máscaras, associado a medidas individuais de proteção. Palavras-chave Coronavirus da sindrome respiratória do Oriente Médio, Sindrome Respiratória aguda grave, Coronavirus, Máscaras, Prevenção 


\section{Introdução}

A partir do reconhecimento do primeiro surto de infecção por COVID 19, registrado em dezembro de 2019, na China continental, em Wuhan, a preocupação com equipamentos de proteção individual (EPI) para a prevenção da propagação da infecção respiratória pelo vírus- SARS-coV-2, via gotículas e ou aerossol, tornou-se um grande desafio frente à pandemia da COVID 19 $19^{1,2}$.

Para assegurar o máximo de segurança contra a transmissão da doença, conforme o Regulamento Sanitário Internacional (RSI), algumas medidas foram adotadas para mitigar os seus efeitos na população e para reduzir os impactos negativos da doença, devido à elevada morbidade e mortalidade causada pelo SARS-CoV-2. No Brasil, visando à ampliação de ações prioritárias, iniciou-se o processo de elaboração de normas técnicas referentes à utilização de equipamentos de proteção individual para o enfrentamento da pandemia atual, dentre eles a orientação do uso de máscaras cirúrgicas e de proteção ${ }^{3}$.

Nesse período buscou-se também ampliar o conhecimento em relação ao tipo de máscara que deverá ser disponibilizada para a contenção da propagação da pandemia. As chamadas máscaras cirúrgicas protegem contra agentes infecciosos transmissíveis por gotículas, são de uso individual, descartáveis, com nível de filtração bacteriana superior a $98 \%$, conforme recomenda a Norma Europeia NE-14683. As máscaras cirúrgicas devem conter 3 camadas independentes de "spunbond-meltblown-spunbond" (não tecido para artigos de uso odonto-médico-hospitalar), seguindo a orientação da ABNT NBR $15.052^{4}$.

O outro tipo, é designado máscara de proteção, conhecido como respirador particulado N95, PFF2 ou equivalentes, segue a Norma Europeia EN-149:2991. Este respirador amplia o seu espectro na filtração de aerossóis, e a depender das normas e legislação de cada país, poderá ser reutilizado. Confere proteção contra agentes infecciosos transmissíveis por gotículas e previnem a inalação de agentes infecciosos propagados por via aérea (aerossóis), partículas de dimensões iguais ou inferiores a $5 \mu \mathrm{m}^{4,5}$.

No momento atual, devido à escassez de insumos a nível global, principalmente de máscaras cirúrgicas, foi recomendado pela Organização Mundial da Saúde o uso de máscara para a população. No entanto, quais as evidências disponíveis sobre a eficácia das máscaras faciais padrão (TNT) em comparação com outras máscaras faciais ou o não uso de máscaras faciais na preven- ção de doenças respiratórias causadas por coronavírus (MERS CoV, SARS-CoV e SARS-CoV-2) na população em geral?

As máscaras a serem utilizadas por não profissionais são máscaras de tecido compostas por duas camadas, fabricadas com "spunbond", um tipo de TNT que não possui a capacidade de filtração para ambientes odonto-médico-hospitalares, não sendo seu uso aconselhável, portanto, por profissionais da saúde, sendo orientada a sua utilização para uso não profissional, com o objetivo de proteger contra doenças respiratórias, entre elas a COVID196.

Desta forma, esta revisão sistemática teve o objetivo de avaliar e sintetizar evidências científicas disponíveis sobre a eficácia das máscaras faciais na prevenção de doenças respiratórias causadas por MERS CoV, SARS-CoV e SARS-CoV-2 na população em geral.

\section{Métodos}

\section{Critérios de inclusão}

Estudos (revisões sistemáticas: meta-análises, ensaio clínicos, randomizados ou não (humanos), observacionais) que avaliaram o uso de máscaras faciais de TNT comparados com outros tipos de máscaras ou o não uso de máscaras na população geral para prevenção de infecções respiratórias virais (MERS-CoV, SARS-CoV e SARS-CoV-2) foram considerados elegíveis para inclusão na presente revisão rápida. Não foram aplicados filtros relacionados a data, idioma ou status de publicação, conforme Quadro 1.

\section{Critérios de exclusão}

Foram excluídos os estudos que avaliaram profissionais da saúde, máscaras cirúrgicas ou respiradores N95 ou PFF2, bem como estudos pré-clínicos, laboratoriais, de avaliação de tecnologias em saúde, editoriais, comentários, artigos de opinião e revisões narrativas, conforme Quadro 2.

\section{Fontes de informação e estratégias de busca}

As buscas da literatura foram realizadas nas bases de dados Medline (via Pubmed), Embase, Cinahl, The Cochrane Library, TripDatabase. As estratégias de busca utioizaram descritores e termos livres para as palavras "Coronavírus", "Covid 19, "Síndrome respiratória aguda grave" e "Más- 
Quadro 1. Critérios de Inclusão dos Estudos

\begin{tabular}{|l|l|}
\hline \multicolumn{1}{|c|}{ População } & \multicolumn{1}{c|}{ Abrange população geral } \\
\hline Intervenção & Abrange máscara facial TNT \\
\hline Desfecho & $\begin{array}{l}\text { Abrange eficácia na proteção de doenças respiratórias por MERS-CoV/SARS-CoV/SARS-CoV-2 } \\
\text { Abrange contaminação por doenças respiratórias do tipo MERS-CoV/SARS-CoV/SARS-CoV-2 }\end{array}$ \\
\hline $\begin{array}{l}\text { Tipo de } \\
\text { Estudo }\end{array}$ & $\begin{array}{l}\text { Revisão Sistemática: meta-análises } \\
\text { Ensaio clínicos, randomizados ou não (humanos) } \\
\text { Observacionais }\end{array}$ \\
\hline Período & Qualquer período \\
\hline
\end{tabular}

Quadro 2. Critérios de Exclusão dos Estudos.

\begin{tabular}{|l|l|}
\hline População & $\begin{array}{l}\text { Não abrange população geral } \\
\text { Abrange Profissionais de saúde }\end{array}$ \\
\hline Intervenção & $\begin{array}{l}\text { Não abrange máscara facial TNT } \\
\text { Abrange máscara cirúrgica e N95 }\end{array}$ \\
\hline Desfecho & $\begin{array}{l}\text { Não abrange eficácia na proteção de doenças respiratórias por MERS-CoV/SARS-CoV/SARS-CoV-2 } \\
\text { Não abrange contaminação por doenças respiratórias do tipo MERS-CoV/SARS-CoV/SARS-CoV-2 } \\
\text { Abrange H1N1 }\end{array}$ \\
\hline $\begin{array}{l}\text { Tipo de } \\
\text { Estudo }\end{array}$ & Editoriais; revisões de literatura; laboratoriais, ATS \\
\hline Período & Não se aplica \\
\hline
\end{tabular}

caras". Para a busca complementar utilizou-se Google Acadêmico, Rayyan, medRxive. A busca foi conduzida no dia 02 de abril de 2020, com última atualização feita em 08 de abril de 2020 (Quadro 3).

\section{Resultados}

\section{Seleção dos estudos}

Foram identificados 1.132 estudos nas bases de dados e na busca complementar. Depois de retirar as duplicatas permaneceram 679 , que foram triados por título e resumo. Desse modo, 69 permaneceram e foram submetidos à leitura integral, sendo selecionados 17 artigos. Finalmente 3 estudos atendiam aos critérios de inclusão e exclusão: 1 ensaio clínico randomizado tipo cluster $^{7}$ e 2 revisões sistemáticas ${ }^{8,9}$ (Figura 1).

\section{Ensaio clínico randomizado}

O trabalho de MacIntyre et al. ${ }^{7}$, é ensaio clínico randomizado em cluster, sobre risco de infec- ção por doença respiratória semelhante à gripe em pessoas que compartilham mesmo domicílio de uma pessoa infectada. A intervenção foi uso de máscara em casa comparado ao não uso. Não foi encontrado resultado estatisticamente significativo para uso de máscara e risco de infecção. Contudo, houve resultado significativo para uso de máscara e redução do risco de desenvolver doença respiratória semelhante à influenza.

$\mathrm{Na}$ análise por intenção de tratar, o risco relativo (RR) para doença respiratória clínica foi de [0,61, 95\% IC 0,18 a 2,13], ILI RR [0,32, IC 95\% 0,03 a 3,13] e para infecções virais confirmadas em laboratório foi de RR [0,97, IC95\% de 0,06 a 15,54$]$, os quais foram consistentemente menores no grupo da máscara comparado ao controle, embora não estatisticamente significante. Os vírus foram isolados em 60\% (146/245) dos casos índice. A influenza foi o vírus mais comum isolado de 115 (47\%) casos - influenza A - 100, influenza B -11 e influenza A e B - 4. Outros vírus isolados dos casos índice foram rinovírus, NL63 e C229E. Mais de um vírus foi isolado em 48 (20\%) casos índice, incluindo 17 coinfecções por influenza. 
Quadro 3. Estratégias de busca utilizadas para as respectivas bases de dados e número de achados.

\begin{tabular}{|c|c|c|}
\hline $\begin{array}{c}\text { Base de dados/ } \\
\text { data }\end{array}$ & Estratégia de busca & Resultados \\
\hline $\begin{array}{l}\text { Medline/PubMed } \\
02 / 04 / 2020\end{array}$ & 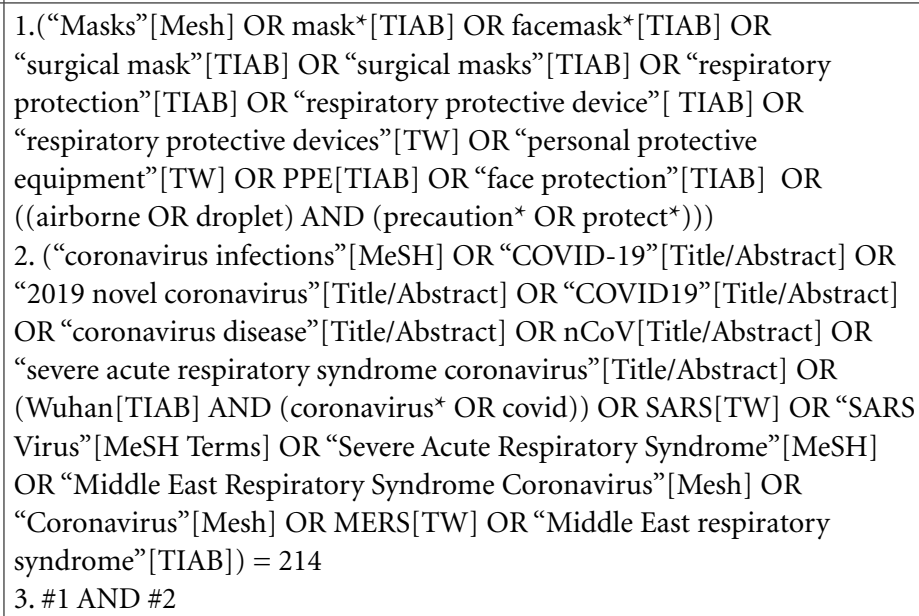 & 400 \\
\hline $\begin{array}{l}\text { Embase/ (Embase. } \\
\text { com) } \\
02 / 04 / 2020\end{array}$ & $\begin{array}{l}\text { 1. 'mask'/exp } \\
\text { 2. mask:ti,ab,kw OR 'face mask':ti,ab,kw OR 'surgical mask':ti,ab,kw OR } \\
\text { 'respiratory protection':ti,ab,kw OR 'face protection':ti,ab,kw } \\
\text { 3. coronavirus infection'/exp OR 'severe acute respiratory syndrome'/exp } \\
\text { OR 'sars-related coronavirus'/exp OR 'middle east respiratory syndrome } \\
\text { coronavirus'/exp } \\
\text { 4. 'covid 19':ti,ab OR '2019 novel coronavirus':ti,ab OR covid19:ti,ab OR } \\
\text { 'coronavirus disease':ti,ab OR ncov:ti,ab OR 'severe acute respiratory } \\
\text { syndrome coronavirus':ti,ab } \\
\text { 5. Wuhan AND (coronavirus:ti,ab,kw OR covid:ti,ab,kw) } \\
\text { 6. sars:ti,ab OR mers:ti,ab OR 'middle east respiratory syndrome':ti,ab } \\
\text { 7. [embase]/lim NOT ([embase]/lim AND [medline]/lim) } \\
\text { 8. \#1 or \#2 } \\
\text { 9. \#3 or \#4 or \#5 or \#6 } \\
\text { 10. \#8 and \#9 } \\
11 . \# 10 \text { and \#7 }\end{array}$ & 81 \\
\hline $\begin{array}{l}\text { Cinahl } \\
02 / 04 / 2020\end{array}$ & 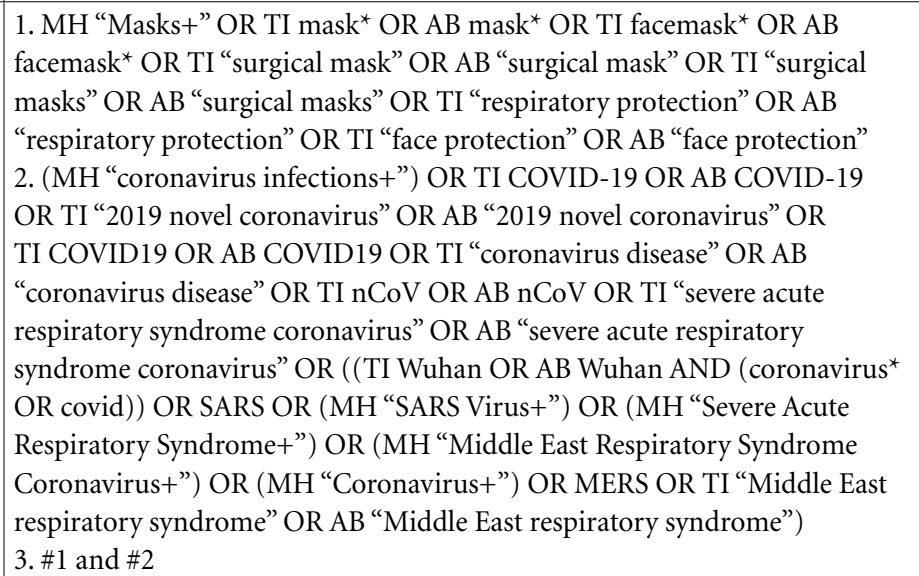 & 117 \\
\hline
\end{tabular}

continua

O risco de viés do estudo foi avaliado através da ferramenta ROB $2.0^{10}$, sendo classificado como incerto. Embora se trate de pesquisa com aparente rigor metodológico, não são fornecidas informações acerca do método de randomização dos participantes da pesquisa. Outro detalhe que chama atenção é o fato de que, associado à utilização de máscaras, foi fornecida informação para 
Quadro 3. Estratégias de busca utilizadas para as respectivas bases de dados e número de achados.

\begin{tabular}{|c|c|c|}
\hline $\begin{array}{c}\text { Base de dados/ } \\
\text { data }\end{array}$ & Estratégia de busca & Resultados \\
\hline $\begin{array}{l}\text { The Cochrane } \\
\text { Library } \\
02 / 04 / 2020\end{array}$ & 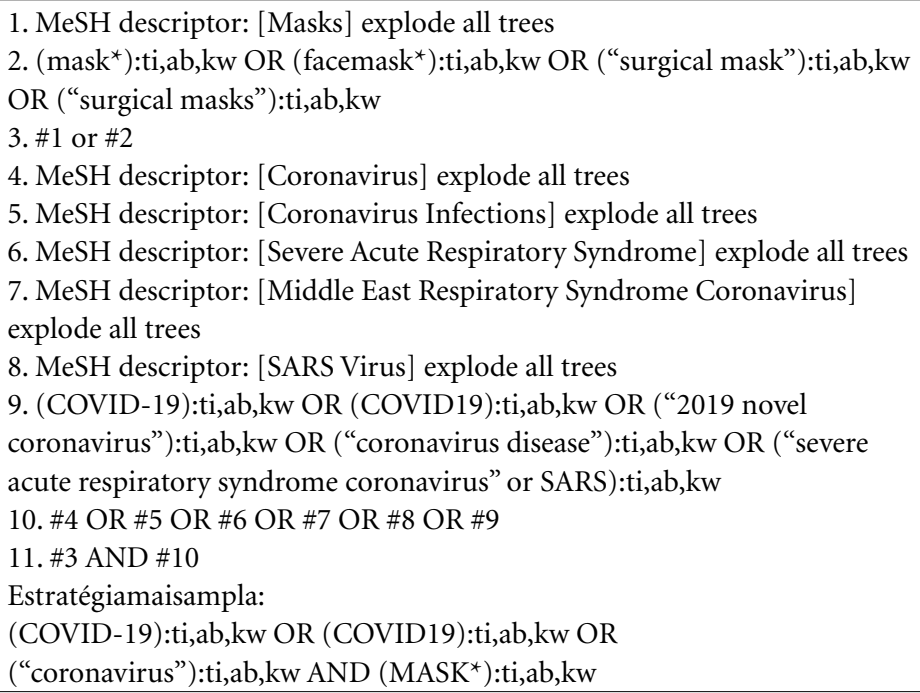 & 42 \\
\hline Lilacs & $\left(\right.$ mask $^{\star}$ or mascara ${ }^{\star}$ or barbijo $\left.{ }^{\star}\right)$ and (covid or coronavirus or sars) & 5 \\
\hline Trip 02/4/2020 & $\left(\right.$ mask $^{\star}$ or facemask $\left.{ }^{\star}\right)$ and (coronavirus or covid) & 126 \\
\hline $\begin{array}{l}\text { Rayyan } \\
\text { [Public] 2020-03-28: } \\
\text { Review containing } \\
\text { the “COVID-19 } \\
\text { Open Research } \\
\text { Dataset” metadata } \\
\text { (45774 articles) } \\
\text { 02/04/2020 }\end{array}$ & $\begin{array}{l}\text { Busca por: Mask } \\
\text { Seleção prévia por títulos eliminado inconsistências }\end{array}$ & 354 \\
\hline $\begin{array}{l}\text { Google Acadêmico } \\
08 / 04 / 2020\end{array}$ & $\left(\right.$ mask $^{\star}$ or facemask $\left.{ }^{*}\right)$ and (coronavirus or covid) & 4 \\
\hline medRxiv & $\left(\right.$ mask $^{\star}$ or facemask $\left.{ }^{\star}\right)$ and (coronavirus or covid & 3 \\
\hline
\end{tabular}

lavagem frequente das mãos no grupo intervenção, o que pode ter contribuído para a diferença observada, mesmo que não estatisticamente significante. $\mathrm{O}$ estudo indica um benefício potencial de máscaras médicas para controle da fonte, mas é limitado por amostra pequena e baixas taxas de ataque secundário (SAR) (Quadro 4).

\section{Revisões sistemáticas}

A revisão de Benkouiten et al. ${ }^{8}$ avaliou uso de intervenções não farmacológicas (INF) para a prevenção de infecções do trato respiratório (IRAs) durante a peregrinação para o Hajj (Mecca). Foram incluídos 17 estudos, sendo realizada análise descritiva dos resultados encontrados. Várias medidas de prevenção de infecções do tra- to respiratório foram analisadas, dentre elas, uso de máscaras faciais. Os resultados quanto à eficácia das máscaras para prevenção de infecções do trato respiratório foram contraditórios. A revisão foi considerada de baixa qualidade, segundo a ferramenta aplicada (AMSTAR 2) ${ }^{11}$.

A eficácia das máscaras faciais na prevenção da transmissão de doenças respiratórias semelhantes à gripe e IRAs depende de vários fatores, como uso de forma consistente e rigoroso de máscaras e a lavagem das mãos. Embora a maioria dos estudos tenha apontado que uso de máscaras estava associado a risco reduzido de síndrome respiratória aguda grave, nenhum dos estudos estabeleceu relação conclusiva entre uso da máscara e proteção contra infecção por doenças respiratórias semelhantes à gripe. Con- 


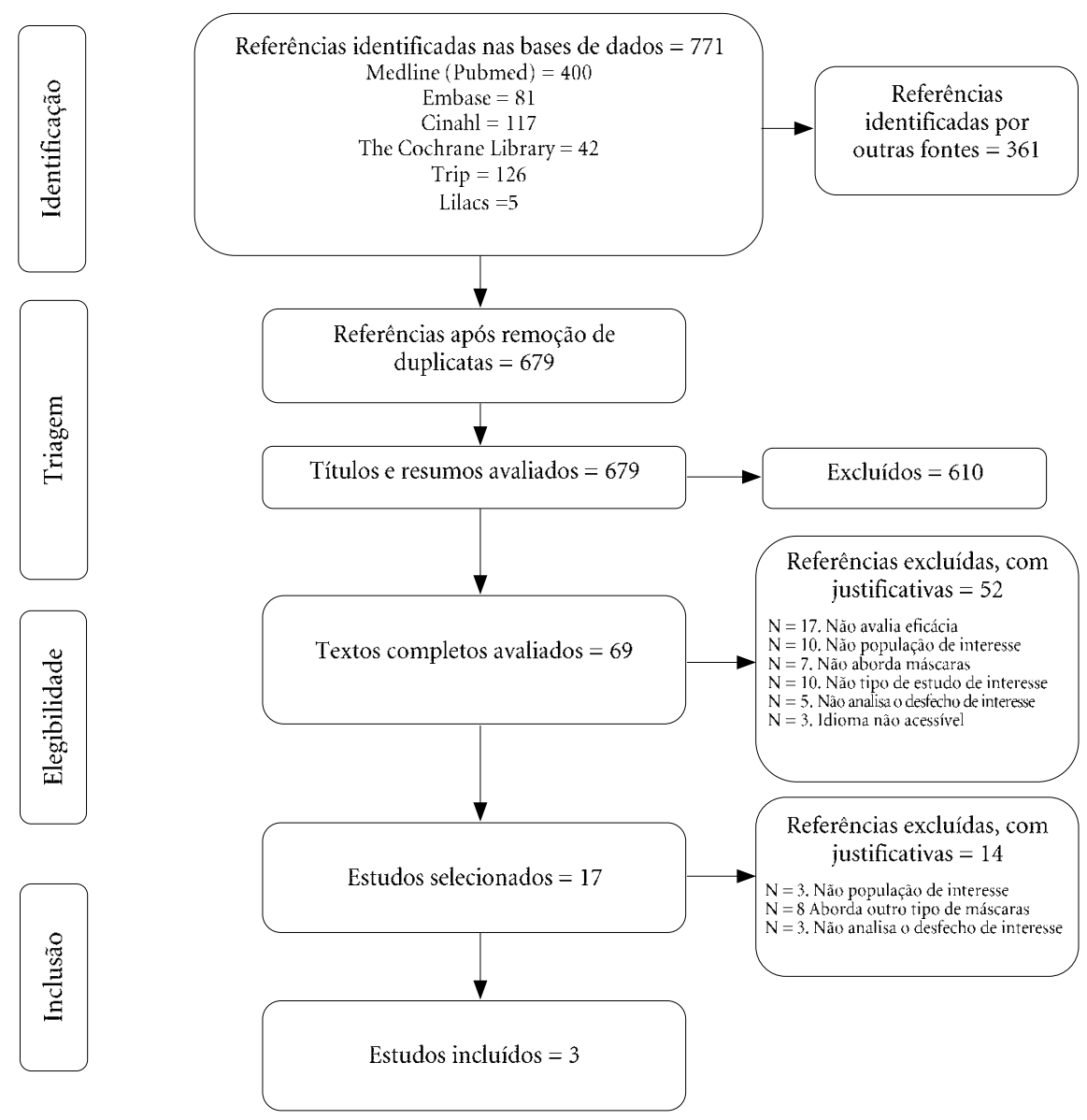

Figura 1. Fluxograma do processo de busca e seleção dos estudos.

tudo, os autores da revisão recomendam uso de máscaras faciais desde o início da peregrinação, de forma consistente e correta, embora os estudos disponíveis não forneçam fortes evidências de sua eficácia na prevenção da infecção respiratória viral.

A revisão sistemática de Liang et al. ${ }^{9}$ avaliou associação do uso da máscara com infecção por vírus respiratório coronavírus da síndrome respiratória aguda grave (SARS-CoV), vírus influenza, vírus H1N1 e SARS-CoV-2. Os 21 estudos incluídos que relataram eficácia do uso de máscaras incluíram 8.686 participantes. Em geral, máscaras são eficazes na prevenção da propagação de vírus respiratórios: depois de usar máscara, a chance de contrair infecção respiratória viral foi significativamente reduzida em 35\% [OR de 0,35. IC $\left.95 \%=0,24-0,51, \mathrm{I}^{2}=60 \%\right]$. No subgrupo de profissionais de saúde o efeito protetor foi mais evidente, com chance de contrair infecção respiratória viral diminuída em 20\% [OR de 0,20. IC $\left.95 \%=0,11-0,37, \mathrm{I}^{2}=59 \%\right]$. Em estudo que investigou a COVID19, a redução da chance de contrair doença foi de 4\% [OR de 0,04. IC 95\% = 0,00-0,60]. No subgrupo de não profissionais de saúde, foi encontrado efeito protetor, com chance de contrair doença 53\% menor [OR agrupado de 0,53 . IC $95 \%=0,36-0,79, \mathrm{I}^{2}=45 \%$ ]. Uma descrição mais detalhada encontrou efeitos significativos no subgrupo doméstico, com $60 \%$ menos chance de contrariar infecção respiratória viral $\left[\mathrm{OR}=0,60\right.$. IC95\% $\left.=0,37-0,97, \mathrm{I}^{2}=31 \%\right] \mathrm{e}$ subgrupo não doméstico, com $44 \%$ menos chance $\left[\mathrm{OR}=0,44\right.$, IC 95\% =0,33-0,59, $\left.\mathrm{I}^{2}=54 \%\right]$. Um estudo incluiu trabalhadores da saúde e familiares de pacientes, com chance $74 \%$ menor de contrair doenças respiratórias virais [OR de 0,74 . IC 95\%: 0,29-1,90]. Por localizações geográficas, 


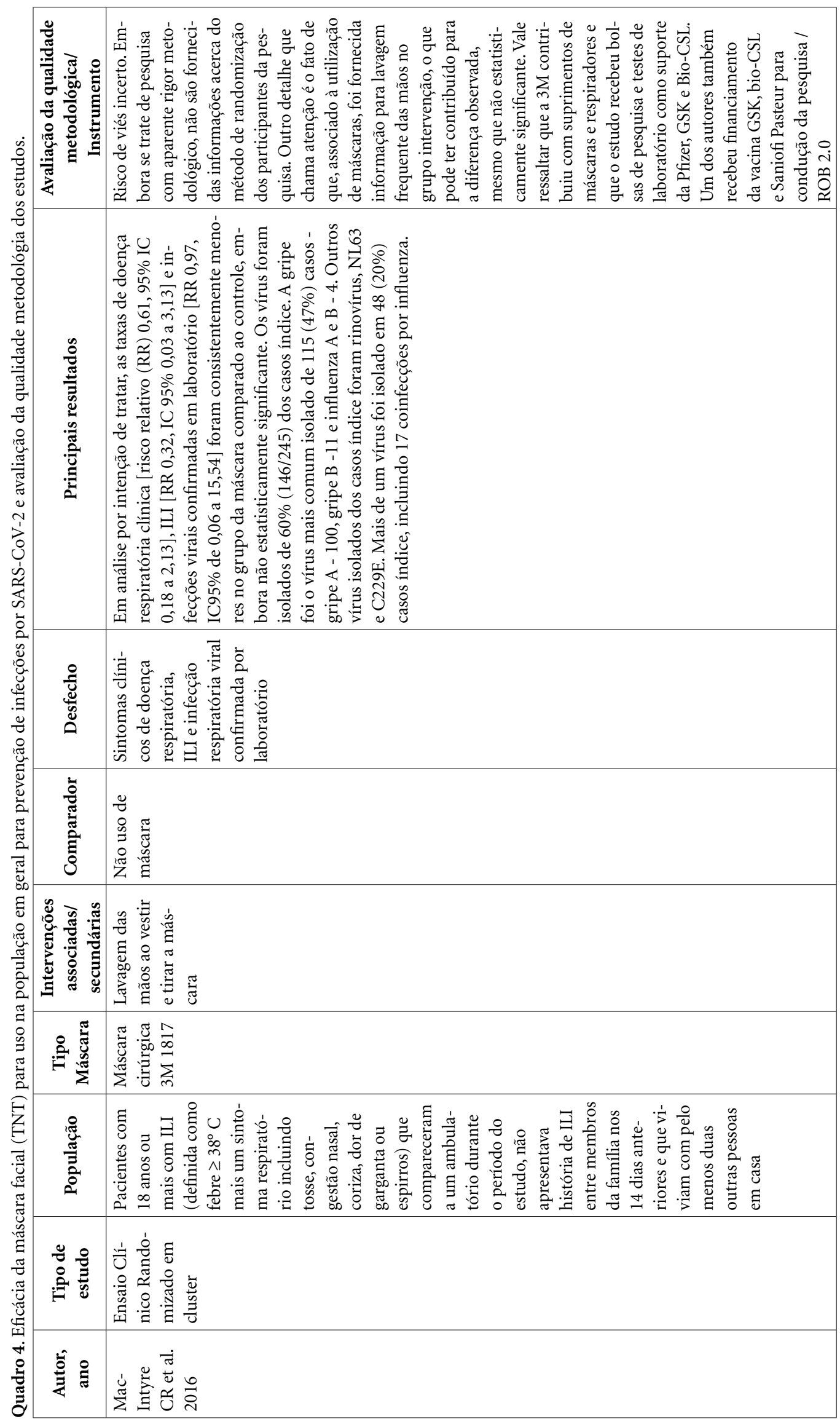




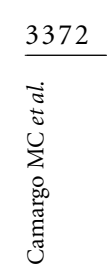

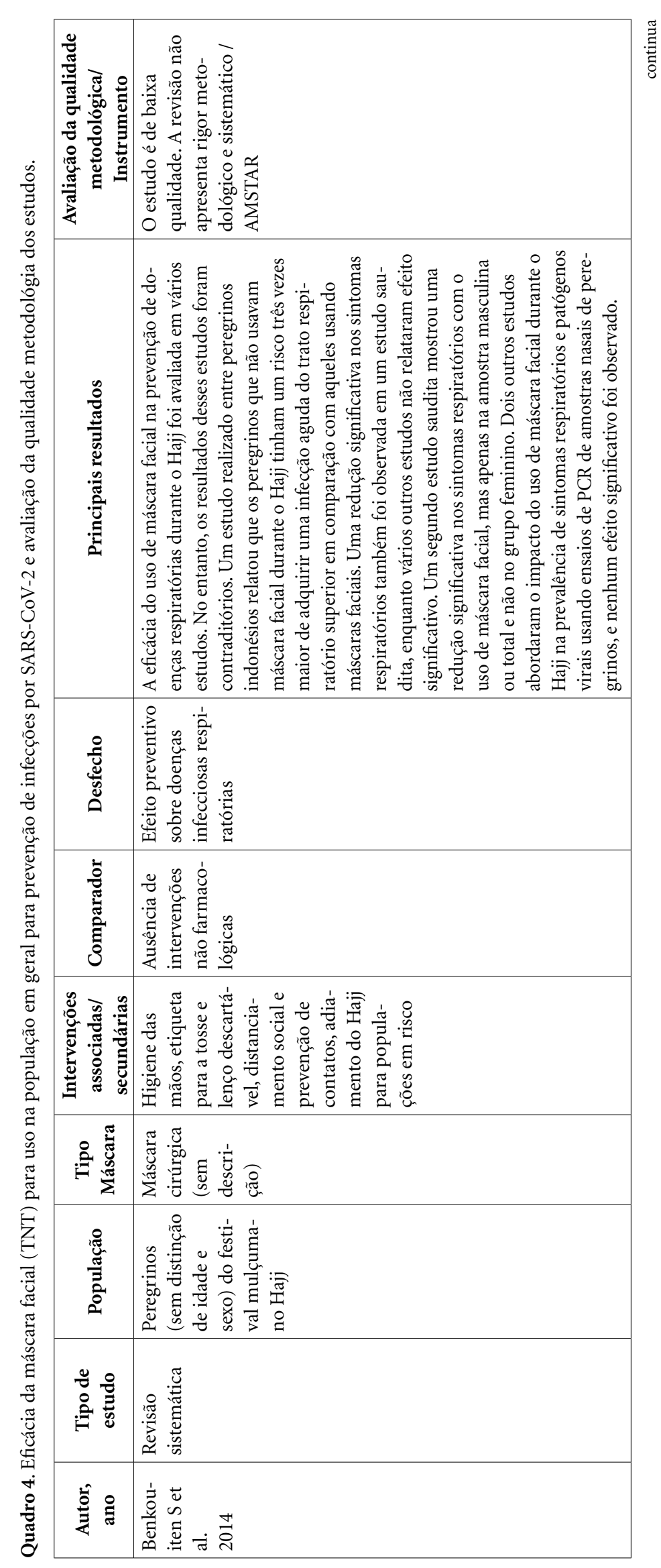




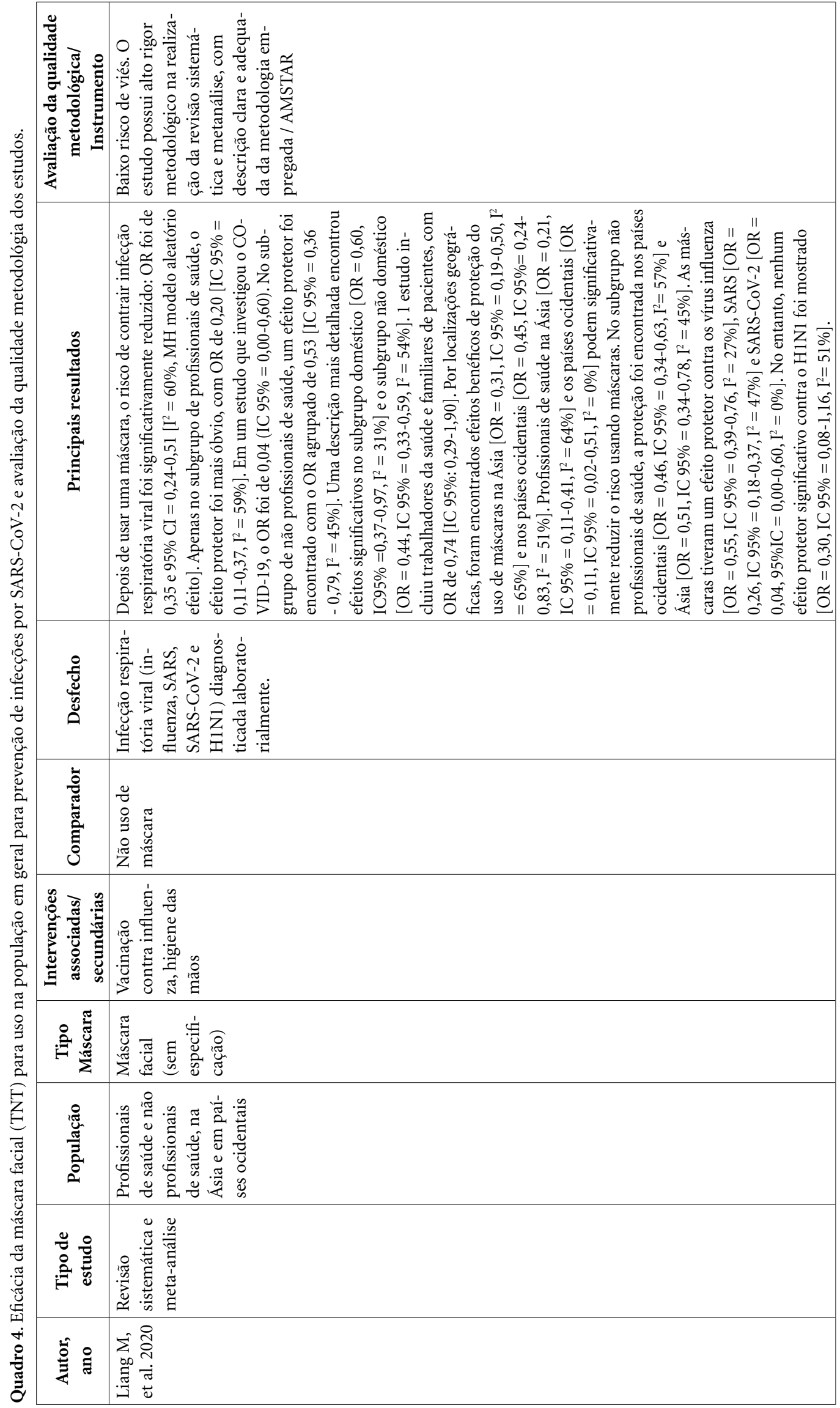


foram encontrados efeitos benéficos de proteção do uso de máscaras na Ásia ( $31 \%$ menos chance) $\left[\mathrm{OR}=0,31\right.$, IC $\left.95 \%=0,19-0,50, \mathrm{I}^{2}=65 \%\right]$ e países ocidentais (chance $45 \%$ menor) [OR $=0,45$, IC $\left.95 \%=0,24-0,83, \mathrm{I}^{2}=51 \%\right]$. Profissionais de saúde na Ásia ( $21 \%$ menos chance) [OR $=0,21$, IC $\left.95 \%=0,11-0,41, \mathrm{I}^{2}=64 \%\right]$ e países ocidentais ( $11 \%$ menos chance) $[\mathrm{OR}=0,11$, IC $95 \%=0,02$ $\left.0,51, \mathrm{I}^{2}=0 \%\right]$ podem significativamente reduzir risco usando máscaras. No subgrupo não profissionais de saúde, proteção foi encontrada nos países ocidentais (chance $46 \%$ menor) [OR $=0,46$, IC $\left.95 \%=0,34-0,63, \mathrm{I}^{2}=57 \%\right]$ e Ásia (51\% menos chance) $\left[\mathrm{OR}=0,51\right.$, IC $95 \%=0,34-0,78, \mathrm{I}^{2}$ $=45 \%]$. Máscaras tiveram efeito protetor contra vírus influenza (chance $55 \%$ menor) $[\mathrm{OR}=0,55$, IC $\left.95 \%=0,39-0,76, \mathrm{I}^{2}=27 \%\right]$, SARS (chance $26 \%$ menor) $\left[\mathrm{OR}=0,26\right.$, IC $95 \%=0,18-0,37, \mathrm{I}^{2}$ $=47 \%]$ e SARS-CoV-2 ( $4 \%$ menor) $[\mathrm{OR}=0,04$, $\left.95 \% \mathrm{IC}=0,00-0,60, \mathrm{I}^{2}=0 \%\right]$. No entanto, nenhum efeito protetor significativo contra $\mathrm{H} 1 \mathrm{~N} 1$ foi mostrado [OR $=0,30$, IC $95 \%=0,08-1,16, \mathrm{I}^{2}=$ 51\%]. A avaliação realizada através do AMSTAR $2^{11}$ indica alta qualidade desta revisão sistemática.

\section{Discussão}

\section{Síntese das evidências}

Os resultados quanto à eficácia das máscaras foram contraditórios, inicialmente devido à falta de definição do tipo de máscaras utilizadas nos estudos: a maioria utiliza apenas o termo genérico "máscaras" ou "máscaras faciais". Houve, entretanto, resultado significativo para o uso de máscara facial TNT e a redução do risco de desenvolver doença respiratória semelhante à gripe. O uso de máscaras faciais padrão TNT tem sido apontado como importante barreira na disseminação de gotículas e aerossol frente à COVID 19, mesmo com suas limitações de não possuir a capacidade de filtração para ambientes odontomédico-hospitalares ${ }^{4}$.

Outro elemento fundamental a ser considerado, o uso de máscara não reduz ou substitui a necessidade das medidas de higiene preconizadas, principalmente a lavagem das mãos, e a manutenção do distanciamento de mais de 1 (um) metro entre as pessoas. Além desse ponto, para que a população tenha acesso a máscara facial TNT, é necessário que seja uma máscara de baixo custo. Devem ainda ser avaliados o tipo de ma- terial, a gramatura recomendada pela ANVISA de $20-40 \mathrm{~g} / \mathrm{m}^{2}$, sendo assegurado que o produto manufaturado tenha 3 camas: uma camada de tecido não impermeável na parte frontal, tecido respirável no meio e um tecido de algodão na parte em contato com a superfície do rosto ${ }^{5}$.

\section{Conclusão}

As evidências consideradas nesta revisão sistemática apontam para um benefício potencial das máscaras faciais padrão TNT para a prevenção da doença COVID 19 e seu uso na população em geral, mas é limitada pelo pequeno número de artigos disponíveis e a baixa qualidade metodológica de dois dos estudos, apresentando uma série de vieses que comprometem os estudos avaliados. Para o cenário atual de pandemia da doença COVID 19 recomenda-se que a população siga a recomendação atual da Organização Mundial de Saúde (OMS) ou as diretrizes locais; educação sobre o uso adequado de máscaras; bem como a utilização de máscaras faciais padrão TNT e ou outros tipos de máscaras como método adjuvante, associado a ações como a higiene das mãos e outras medidas individuais de proteção. Contudo, estudos adicionais que investiguem a eficácia comparativa do uso das máscaras faciais TNT e ou outros tipos de máscaras para a população em geral são necessários, de forma a contribuir para orientar medidas preventivas frente à epidemia da COVID 19.

\section{Fortalezas e limitações da revisão sistemática}

Esta revisão sistemática tem precauções metodológicas, como pesquisa em bancos de dados importantes e a avaliação da qualidade metodológica dos estudos incluídos. Contudo, alguns fatores foram considerados limitantes, como a escassez de artigos e a baixa qualidade metodológica de dois dos estudos selecionados. Não obstante as evidências consideradas nesta revisão sistemática apontem para um benefício potencial das máscaras faciais padrão TNT para a prevenção da doença COVID 19 e seu uso na população em geral, não é possível estabelecer resultado conclusivo, devido à existência de poucos estudos primários que abordem máscaras faciais TNT, e ou outros tipos de máscaras, comparadas com o não uso de máscaras para a prevenção da SARScoV-2 na população em geral. 


\section{Colaboradores}

Os autores MC Camargo, MS Martinez-Silveira, AA Lima participaram da elaboração do projeto, coleta de dados, análise dos dados e redação do artigo, BP Bastos, DL Santos, SEC Mota participaram da análise dos dados e redação do artigo, RB Silva, IP Toledo participaram da revisão artigo.

\section{Referências}

1. Li Q, Guan X, Wu P, Wang X, Zhou L, Tong Y, Ren R, Leung KSM, Lau EHY, Wong JY, Xing X, Xiang N, Wu Y, Li C, Chen Q, Li D, Liu T, Zhao J, Liu M, Tu W, Chen C, Jin L, Yang R, Wang Q, Zhou S, Wang R, Liu H, Luo Y, Liu Y, Shao G, Li H, Tao Z, Yang Y, Deng Z, Liu B, Ma Z, Zhang Y, Shi G, Lam TTY, Wu JT, Gao GF, Cowling BJ, Yang B, Leung GM, Feng Z. Early Transmission dynamics in Wuhan, China, of novel coronavirus-infected pneumonia. N Engl J Med 2020; 382(13):1199-1207.

2. Zhu N, Zhang D, Wang W, Li X, Yang B, Song J, Zhao X, Huang B, Shi W, Lu R, Niu P, Zhan F, Ma X, Wang D, Xu W, Wu G, Gao GF, Tan W, China Novel Coronavirus Investigating and Research Team. A Novel Coronavirus from Patients with Pneumonia in China, 2019. N Engl J Med 2020; 382(8):727-733.

3. Organização Mundial da Saúde (OMS). WHA58.3, Revision of the International Health Regulations. Genebra: OMS; 2005.

4. Agência Nacional de Vigilância Sanitária (Anvisa). Nota técnica GVIMS/GGTES/ANVISA, no 04/2020. Orientações para serviços de saúde: medidas de prevenção e controle que devem ser adotadas durante a assistência aos casos suspeitos ou confirmados de infecção pelo novo coronavírus (sars-cov-2) (atualizada em 31/03/2020). Brasília: Anvisa; 2020.

5. Agência Nacional de Vigilância Sanitária (Anvisa). Resolução de Diretoria Colegiada - RDC No 356, de 23 de março de 2020. Dispõe, de forma extraordinária e temporária, sobre os requisitos para a fabricação, importação e aquisição de dispositivos médicos identificados como prioritários para uso em serviços de saúde, em virtude da emergência de saúde pública internacional relacionada ao SARS-CoV-2. Diário Oficial da União 2020; 23 mar.

6. World Health Organization (WHO). Advice on the use of masks in the context of COVID-19: interim guidance, 6 April 2020. Geneva: WHO; 2020. [Contract No.: WHO/2019-nCov/IPC_Masks/2020.3]

7. MacIntyre CR, Zhang Y, Chughtai AA, Seale H, Zhang D, Chu Y, Zhang H, RahmanB, Wang Q. Cluster randomised controlled trial to examine medical mask use as source control for people with respiratory illness. BMJ Open 2016; 6(12):e012330.

8. Benkouiten S, Brouqui P, Gautret P. Non-pharmaceutical interventions for the prevention of respiratory tract infections during Hajj pilgrimage. Travel Med Infect Dis 2014; 12(5):429-442.

9. Liang M, Gao L, Cheng C, Zhou Q, Uy JP, Heiner K, Sun C. Efficacy of face mask in preventing respiratory virus transmission: a systematic review and meta-analysis. RedRxiv 2020; [acessado 2020 Maio 06]. Disponível em: https://doi.org/10.1101/2020.04.03.20051649

10. Sterne JAC, Savovic J, Page MJ, Elbers RG, Blencowe NS, Boutron I, Cates CJ, Cheng HY, Corbett MS, Eldridge SM, Emberson JR, Hernán MA, Hopewell S, Hróbjartsson A, Junqueira DR, Jüni P, Kirkham JJ, Lasserson T, Li T, McAleenan A, Reeves BC, Shepperd S, Shrier I, Stewart LA, Tilling K, White IR, Whiting $\mathrm{PF}$, Higgins JPT. RoB 2: a revised tool for assessing risk of bias in randomised trials. BMJ 2019; 366:14898. 
11. Shea BJ, Reeves BC, Wells G, Thuku M, Hamel C, Moran J, Moher D, Tugwell P, Welch V, Kristjansson E, Henry DA. AMSTAR 2: a critical appraisal tool for systematic reviews that include randomised or non-randomised studies of healthcare interventions, or both. BMJ 2017; 358:j4008.

Artigo apresentado em 07/05/2020

Aprovado em 07/05/2020

Versão final apresentada em 09/05/2020 